\title{
BOUNDS FOR THE DISTRIBUTION OF TWO DIMENSIONAL BINARY SCAN STATISTICS
}

\author{
Michael V. Boutsikas and Markos V. Koutras \\ Department of Statistics and Insurance Science, University of Piraeus, Greece.
}

\begin{abstract}
In the present article we develop some efficient bounds for the distribution function of a two dimensional scan statistic defined on a (double) sequence of iid binary trials. The methodology employed here takes advantage of the connection between the scan statistic problem and a equivalent reliability structure and exploits appropriate techniques of reliability theory to establish tractable bounds for the distribution of the statistic of interest. An asymptotic result is established and a numerical study is carried out to investigate the efficiency of the suggested bounds.

Keywords and phrases: scan statistics, reliability bounds, covariance bounds, two-dimensional$r$-within-consecutive- $k_{1} \times k_{2}$-out-of- $n_{1} \times n_{2}$ system.
\end{abstract}

\section{Introduction}

Assume that a two dimensional rectangular region $R=\left[0, L_{1}\right] \times\left[0, L_{2}\right]$ is observed and our interest is focused on the patterns in which a certain event $\mathcal{E}$ occurs in $R$. let $n_{1}, n_{2}$ be two positive integers and define $h_{i}=L_{i} / n_{i}, i=1,2$. Furthermore, assume that $n_{1}, n_{2}$ are large enough so that in each of the $n_{1} \times n_{2}$ rectangular subregions

$$
R_{i j}=\left[(i-1) h_{1}, i h_{1}\right] \times\left[(j-1) h_{2}, i h_{2}\right], i=1,2, \ldots, n_{1}, j=1,2, \ldots, n_{2}
$$

the event $\mathcal{E}$ may occur only once or does not occur at all. A question that comes in naturally within this setup is whether reasonable criteria providing evidence of clustering of the occurrences of $\mathcal{E}$ over $R$ could be established.

In order to establish a probabilistic model, let us introduce the Bernoulli r.v.'s $X_{i j}, i=1,2, \ldots, n_{1}, j=$ $1,2, \ldots, n_{2}$ indicating the occurrence $\left(X_{i j}=1\right)$ or non-occurrence $\left(X_{i j}=0\right)$ of the event $\mathcal{E}$ in subregion $R_{i j}$. We assume that $X_{i j}$ are iid r.v.'s with success probabilities

$$
q=P\left(X_{i j}=1\right)=1-P\left(X_{i j}=0\right)=1-p .
$$

For $1 \leq a \leq n_{1}-k_{1}+1,1 \leq b \leq n_{2}-k_{2}+1$ we define

$$
S(a, b)=\sum_{i=a}^{a+k_{1}-1} \sum_{j=b}^{b+k_{2}-1} X_{i j}
$$

to be the number of events in a rectangular region comprised of $k_{1} \times k_{2}$ adjacent subregions $R_{i j}, a \leq$ $i \leq a+k_{1}-1, b \leq j \leq b+k_{2}-1\left(k_{1}, k_{2}\right.$ are positive integers). If $S(a, b)$ exceeds a preassigned value $r$ we shall say that (at least) $r$ events have been clustered within the inspected region. The two 
dimensional (binary) discrete scan statistic is then defined as the largest number of events in any of the $\left(n_{1}-k_{1}+1\right)\left(n_{2}-k_{2}+1\right)$ observed regions, i.e.

$$
S=S_{k_{1}, k_{2}, n_{1}, n_{2}}=\max \left\{S(a, b): a=1,2, \ldots, n_{1}-k_{1}+1, b=1,2, \ldots, n_{2}-k_{2}+1\right\} .
$$

It is clear that such a statistic can be exploited for testing the null hypothesis of uniformity against an alternative hypothesis of two dimensional clustering, that results in an increased occurrence of events in square subgrids of the rectangular arrangement of the observations.

Astronomy is probably one of the earliest sciences where the scan statistic was fruitfully used. The search of heavens for clustered galaxies, particles, signals or radiation sources attracted the interest of astronomers from the very beginning. Orford [10] reviews approaches for analyzing cosmic ray data in one and two dimensions, with an extensive discussion of the scan statistic. Two dimensional scans play a critical role in several other scientific areas as well, for example, ecology, computer science, mechanical engineering, biosciences, image analysis, minesfield detection, pattern recognition; for a detailed review see Glaz, Naus and Wallenstein [8] and the references therein.

In addition to such diverse and fascinating applications, the study of scan statistics involves many sophisticated and intricate theoretical methods, for example Poisson and compound Poisson approximations, large deviation theory, Bonferroni-type inequalities, order statistics and spacings, Monte Carlo simulation etc.

The distribution of the scan statistic $S$ can alternatively be expressed as the reliability of a twodimensional $r$-within-consecutive- $k_{1} \times k_{2}$-out-of- $n_{1} \times n_{2}$ system. This system consists of $n_{1} n_{2}$ independent components arranged in a square grid of size $n_{1} \times n_{2}$ and fails if and only if there exists at least one square block of size $k_{1} \times k_{2}\left(1<k_{1} \leq n_{1}, 1<k_{2} \leq n_{2}\right)$ which contains at least $r \geq 2$ failed components. A typical application of this system in electrical engineering comes from the TFT Liquid Crystal Display technology where an increased number of non-functional pixels in fixed size rectangular subgrids of the screen is considered as a criterion for rejecting the produced item.

If $R=R_{r, k_{1}, k_{2}, n_{1}, n_{2}}$ denotes the reliability of a two-dimensional $r$-within-consecutive- $k_{1} \times k_{2}$-outof- $n_{1} \times n_{2}$ system with component failure probabilities $q_{i j}, i=1,2, \ldots, n_{1}, j=1,2, \ldots, n_{2}$, it is clear that $P\left(S_{k_{1}, k_{2}, n_{1}, n_{2}} \leq r-1\right)=R_{r, k_{1}, k_{2}, n_{1}, n_{2}}$. Hence, the determination of the distribution of $S$ is equivalent to the assessment of the reliability of a two-dimensional $r$-within-consecutive- $k_{1} \times k_{2}$-out-of- $n_{1} \times n_{2}$ system. Unfortunately, the exact reliability evaluation of this system is a formidable (or non-feasible) task even for moderate values of $k_{1}, k_{2}$ (cf. Akiba and Yamamoto [1]). Therefore, it seems reasonable to resort to approximations or bounds. Chen and Glaz [5] and Makri and Psillakis [9] used Bonferroni inequalities to establish reliability bounds for this system; Chen and Glaz [5] investigated also several product type approximations for the distribution of the two dimensional scan statistic $S$. Recently, Boutsikas and Koutras [4] employed a finite Markov chain imbedding method to derive accurate approximations for $R_{r, k_{1}, k_{2}, n_{1}, n_{2}}$. For a detailed review of these approaches the interested reader may consult Glaz, Naus and Wallenstein [8].

The purpose of this article is to develop improved (upper and lower) bounds for the distribution of the discrete scan statistic $S$ in the case of independent and identical $X_{i j}$ 's. These bounds are extremely useful especially for the cases where the lower and upper bounds are close to each other therefore giving rise to narrow interval estimates for the unknown exact distribution. The consideration here of the special case of iid Bernoulli trials was for typographical convenience only. It is straightforward to verify that many of the results developed in this article could be easily adjusted to the case where the trials are independent but not necessarily identical. This task is left to the interested reader as an exercise.

The derivation of the new bounds is chiefly couched on the general results provided in a recent publication of the authors of the present article. More specifically, Boutsikas and Koutras [3] introduced several generalizations of well-known reliability bounds based on arbitrary partitions of the family of minimal path or cut sets of a coherent structure. After the introduction of the necessary definitions and notations (Section 2) we proceed at the establishment of product-type upper and lower bounds for the cumulative distribution function of the scan statistic (Section 3). In Section 4 we provide some additional bounds of a different nature (non-product-type) while in Section 5 the asymptotic behavior 
of $S$ is investigated by the aid of the results developed in the previous sections. Finally, in Section 6 , a detailed numerical experimentation is carried out, which elucidates the quality of the suggested bounds.

\section{Definitions and notations}

As already mentioned, the development of bounds for the distribution function of the scan statistic $S=S_{k_{1}, k_{2}, n_{1}, n_{2}}$ will be carried out by considering the equivalent two-dimensional $r$-within-consecutive$k_{1} \times k_{2}$-out-of- $n_{1} \times n_{2}$ system, bounding its reliability $R=R_{r, k_{1}, k_{2}, n_{1}, n_{2}}$ from above and below and subsequently using the transition formula $P(S<r)=R$. We shall introduce now the necessary notations for the presentation that will follow. Let $I=\left\{(i, j): i=1,2, \ldots, n_{1}, j=1,2, \ldots, n_{2}\right\}$ be the collection of system's components (equipped with the lexicographic order) and denote by $X_{i j}^{\prime}=1-X_{i j}$, the indicator r.v.'s describing the components' state i.e. $X_{i j}^{\prime}=1$ if component $(i, j)$ is functioning and $X_{i j}^{\prime}=0$ if component $(i, j)$ has failed. Then, the failure probabilities $q$ of system's components may be expressed as

$$
q=P\left(X_{i j}^{\prime}=0\right)=P\left(X_{i j}=1\right)
$$

In order to describe the minimal cut sets of the system we set $N_{1}=n_{1}-k_{1}+1, N_{2}=n_{2}-k_{2}+1$ and introduce the sets

$$
A_{i j}=\left\{(a, b), a=i, i+1, \ldots, i+k_{1}-1, b=j, j+1, \ldots, j+k_{2}-1\right\}, i, j \geq 1 .
$$

By the definition of the system, its minimal cut sets $C$ are all the subsets of $A_{i j}, i=1,2, \ldots, N_{1}, j=$ $1,2, \ldots, N_{2}$ with cardinality $|C|=r$. Therefore, the family $\mathbb{C}$ of all minimal cut sets can be expressed as

$$
\mathbb{C}=\bigcup_{i=1}^{N_{1}} \bigcup_{j=1}^{N_{2}} \mathbb{A}_{i j} \quad \text { where } \mathbb{A}_{i j}=\left\{C: C \subseteq A_{i j} \text { and }|C|=r\right\} .
$$

A more convenient description of $\mathbb{C}$ is achieved by considering the disjoint families of cut sets

$$
\begin{aligned}
\mathbb{C}_{i j} & =\mathbb{A}_{i j} \backslash\left(\mathbb{A}_{i+1, j} \cup \mathbb{A}_{i, j+1}\right), i<N_{1} \text { and } j<N_{2}, \\
\mathbb{C}_{i, N_{2}} & =\mathbb{A}_{i, N_{2}} \backslash \mathbb{A}_{i+1, N_{2}}, i<N_{1}, \mathbb{C}_{N_{1}, j}=\mathbb{A}_{N_{1}, j} \backslash \mathbb{A}_{N_{1}, j+1}, j<N_{2}, \quad \mathbb{C}_{N_{1}, N_{2}}=\mathbb{A}_{N_{1}, N_{2}} .
\end{aligned}
$$

and observing that the next equality is true as well

$$
\mathbb{C}=\bigcup_{i=1}^{N_{1}} \bigcup_{j=1}^{N_{2}} \mathbb{C}_{i j}
$$

If $\mathbb{A}$ is a collection of subsets of $I$, the symbol $R(\mathbb{A})$ will denote the reliability of a system which has as cut sets all the elements of $\mathbb{A}$, that is

$$
R(\mathbb{A})=E\left(\prod_{A \in \mathbb{A}}\left(1-\prod_{(i, j) \in A}\left(1-X_{i j}^{\prime}\right)\right)\right) .
$$

Finally, we shall use the notation

$$
F_{i, m}^{c}=\sum_{x=\max \{i, 0\}}^{m}\left(\begin{array}{c}
m \\
x
\end{array}\right) q^{x} p^{m-x}
$$

for the tail probabilities of the binomial distribution. 


\section{Product-type bounds}

One of the earliest approaches to the problem of developing product-type reliability bounds may be attributed to Esary and Proschan [6] who suggested the simple lower bound

$$
L_{E P}=\prod_{C \in \mathbb{C}}\left(1-\prod_{a \in C} q_{a}\right)
$$

(see also Barlow and Proschan [2]). A direct application of this result in the special case of a twodimensional- $r$-within-consecutive- $k_{1} \times k_{2}$-out-of- $n_{1} \times n_{2}$ system yields the next Proposition.

Proposition 1 The cumulative probability $P(S \leq r-1)=R_{r, k_{1}, k_{2}, n_{1}, n_{2}}$ of the scan statistic $S$ is bounded below by

$$
L_{E P}=\left(1-q^{r}\right)^{|\mathbb{C}|}
$$

where

$$
\begin{aligned}
|\mathbb{C}|= & \left(n_{1}-k_{1}\right)\left(n_{2}-k_{2}\right)\left(\left(\begin{array}{c}
k_{1} k_{2} \\
r
\end{array}\right)-\left(\begin{array}{c}
\left(k_{1}-1\right) k_{2} \\
r
\end{array}\right)-\left(\begin{array}{c}
k_{1}\left(k_{2}-1\right) \\
r
\end{array}\right)+\left(\begin{array}{c}
\left(k_{1}-1\right)\left(k_{2}-1\right) \\
r
\end{array}\right)\right) \\
& +\left(n_{1}-k_{1}\right)\left(\left(\begin{array}{c}
k_{1} k_{2} \\
r
\end{array}\right)-\left(\begin{array}{c}
\left(k_{1}-1\right) k_{2} \\
r
\end{array}\right)\right)+\left(n_{2}-k_{2}\right)\left(\left(\begin{array}{c}
k_{1} k_{2} \\
r
\end{array}\right)-\left(\begin{array}{c}
k_{1}\left(k_{2}-1\right) \\
r
\end{array}\right)\right) \\
& +\left(\begin{array}{c}
k_{1} k_{2} \\
r
\end{array}\right)
\end{aligned}
$$

Proof. Observe first that, for each cut set $C \in A_{i j}$ we have

$$
\prod_{(i, j) \in C} q_{i j}=\prod_{(i, j) \in C} q=q^{|C|}=q^{r}
$$

and therefore,

$$
L_{E P}=\prod_{C \in \mathbb{C}}\left(1-q^{r}\right)=\left(1-q^{r}\right)^{|\mathbb{C}|}
$$

The cardinality of $\mathbb{C}$ can be easily evaluated if we notice that, in view of (9),

$$
|\mathbb{C}|=\sum_{i=1}^{N_{1}} \sum_{j=1}^{N_{2}}\left|\mathbb{C}_{i j}\right|
$$

and then proceed to a careful enumeration of the elements of each family $\mathbb{C}_{i j}$.

The lower bound of Proposition 1 is pleasing in its simplicity; however, it succeeds in approximating satisfactorily the exact cumulative probability only for low values of $q$. A better approximation may be established by using the next proposition.

Proposition 2 The cumulative probability $P(S \leq r-1)=R_{r, k_{1}, k_{2}, n_{1}, n_{2}}$ of the scan statistic $S$ is bounded below by

$$
L_{E P}^{(G)}=\left(1-Q_{1}\right)^{\left(n_{1}-k_{1}\right)\left(n_{2}-k_{2}\right)}\left(1-Q_{2}\right)^{n_{1}-k_{1}}\left(1-Q_{3}\right)^{n_{2}-k_{2}}\left(1-Q_{4}\right)
$$

where

$$
\begin{aligned}
& Q_{1}=F_{r, k_{1} k_{2}}^{c}-p^{k_{2}} F_{r,\left(k_{1}-1\right) k_{2}}^{c}-p^{k_{1}} F_{r, k_{1}\left(k_{2}-1\right)}^{c}+p^{k_{1}+k_{2}-1} F_{r,\left(k_{1}-1\right)\left(k_{2}-1\right)}^{c} \\
& Q_{2}=F_{r, k_{1} k_{2}}^{c}-p^{k_{2}} F_{r,\left(k_{1}-1\right) k_{2}}^{c}, Q_{3}=F_{r, k_{1} k_{2}}^{c}-p^{k_{1}} F_{r, k_{1}\left(k_{2}-1\right)}^{c}, Q_{4}=F_{r, k_{1} k_{2}}^{c}
\end{aligned}
$$


Proof. Since $\mathbb{C}_{i j}, i=1,2, \ldots, N_{1}, j=1,2, \ldots, N_{2}$ are disjoint and satisfy (9), they form a partition of the family $\mathbb{C}$ of system's minimal cut sets. As Boutsikas and Koutras [3] have proven (cf. Theorem 2) the quantity

$$
L_{E P}^{(G)}=\prod_{i=1}^{N_{1}} \prod_{j=1}^{N_{2}} R\left(\mathbb{C}_{i j}\right)
$$

will consist a lower bound of system's reliability. We observe next that each $R\left(\mathbb{C}_{i j}\right)$ can be expressed in terms of the tail probabilities of appropriate binomial distributions. More specifically, on introducing the events

$$
W_{i j}=\left[\sum_{a=i}^{i+k_{1}-1} \sum_{b=j}^{j+k_{2}-1} X_{a b} \geq r\right], \quad H_{i j}=\left[\sum_{b=j}^{j+k_{2}-1} X_{i b}=0\right], \quad V_{i j}=\left[\sum_{a=i}^{i+k_{1}-1} X_{a j}=0\right]
$$

and the index sets,

$$
\begin{aligned}
& T_{1}=\left\{(i, j), i=1,2, \ldots, N_{1}-1, j=1,2, \ldots, N_{2}-1\right\}, T_{2}=\left\{\left(i, N_{2}\right), i=1,2, \ldots, N_{1}-1\right\}, \\
& T_{3}=\left\{\left(N_{1}, j\right), j=1,2, \ldots, N_{2}-1\right\}, T_{4}=\left\{\left(N_{1}, N_{2}\right)\right\},
\end{aligned}
$$

we may write

$1-R\left(\mathbb{C}_{i j}\right)=P\left(W_{i j} H_{i j}^{c} V_{i j}^{c}\right)=P\left(W_{i j}\right)-P\left(W_{i j} H_{i j}\right)-P\left(W_{i j} V_{i j}\right)+P\left(W_{i j} H_{i j} V_{i j}\right)=Q_{1}, \quad(i, j) \in T_{1}$,

$1-R\left(\mathbb{C}_{i j}\right)=P\left(W_{i j} H_{i j}^{c}\right)=P\left(W_{i j}\right)-P\left(W_{i j} H_{i j}\right)=Q_{2}, \quad(i, j) \in T_{2}$,

$1-R\left(\mathbb{C}_{i j}\right)=P\left(W_{i j} V_{i j}^{c}\right)=P\left(W_{i j}\right)-P\left(W_{i j} V_{i j}\right)=Q_{3},(i, j) \in T_{3}$,

$1-R\left(\mathbb{C}_{i j}\right)=P\left(W_{i j}\right)=Q_{4},(i, j) \in T_{4}$.

The desired result can now be easily verified by substituting all $R\left(\mathbb{C}_{i j}\right)$ in $(21)$.

So far, the cumulative probabilities of the scan statistic $S$ were lower bounded by computationally tractable bounds. A natural question at this point is whether upper bounds could also be established by exploiting the connection between the scan statistic distribution and the reliability of a two-dimensional $r$-within-consecutive- $k_{1} \times k_{2}$-out-of- $n_{1} \times n_{2}$ system. Unfortunately, should we wish to calculate the Esary and Proschan [6] upper reliability bounds we need to establish first all the minimal path sets of the system. The computational complexity of the last task is huge and therefore such an approach seems quite intricate. Fu and Koutras [7] developed a set of upper reliability bounds which are based on the minimal cut sets of a coherent structure and offer a satisfactory counterpart for Esary and Proschan lower bounds. In order to apply these, one has to consider a certain ordering (arrangement) of system's minimal cut sets and introduce appropriate index sets. If $L_{C}$ denotes the index set associated to minimal cut set $C \in \mathbb{C}$ (for the details on the construction of it see Fu and Koutras [7]) the upper bounds take on the form

$$
L_{F K}=\prod_{C \in \mathbb{C}}\left(1-\left(\prod_{a \in L_{C}} p_{a}\right)\left(\prod_{b \in C} q_{b}\right)\right) .
$$

In the case of a two-dimensional $r$-within-consecutive- $k_{1} \times k_{2}$-out-of- $n_{1} \times n_{2}$ system, a simple arrangement (linearization) of the family $\mathbb{C}$ of minimal cut sets is established as follows: place first (in any order) the $\left|\mathbb{C}_{11}\right|$ cut sets of the family $\mathbb{C}_{11}$, next place (in any order) the $\left|\mathbb{C}_{12}\right|$ cut sets of the family $\mathbb{C}_{12}$, etc. (use lexicographic ordering for the indices $(i, j)$ in $\left.\mathbb{C}_{i j}\right) \ldots$, place at the end (in any order) the $\left|\mathbb{C}_{N_{1} N_{2}}\right|$ cut sets of the family $\mathbb{C}_{N_{1} N_{2}}$. It can be easily ascertained that the family of index sets

$$
\begin{aligned}
L_{C_{i}}= & \left(\left\{(u, v): u=\max \left\{a-k_{1}+1,1\right\}, \ldots, a+k_{1}-2,\right.\right. \\
& \left.v=\max \left\{b-k_{2}+1,1\right\}, \ldots, \min \left\{b+2 k_{2}-2, n_{2}\right\}\right\} \\
& \left.\cup\left\{\left(a+k_{1}-1, v\right): v=\max \left\{b-k_{2}+1,1\right\}, \ldots, b+k_{2}-1\right\}\right) \backslash C_{i}, \quad\left(a, b: C_{i} \in \mathbb{C}_{a b}\right),
\end{aligned}
$$


$i=2,3, \ldots,|\mathbb{C}|, L_{C_{1}}=\emptyset$, satisfy the conditions required in the Fu and Koutras [7] approach. Thus in the iid case we get the upper bound

$$
U_{F K}=\prod_{j=1}^{|\mathbb{C}|}\left(1-p^{\left|L_{C_{j}}\right|} q^{r}\right)
$$

and taking into account that

$$
\left|L_{C_{j}}\right| \leq\left(2 k_{1}-2\right)\left(3 k_{2}-2\right)+2 k_{2}-1-r,
$$

we easily arrive at the following result.

Proposition 3 The cumulative probability $P(S \leq r-1)=R_{r, k_{1}, k_{2}, n_{1}, n_{2}}$ of the scan statistic $S$ is bounded above by

$$
U_{F K}=\left(1-p^{\left(2 k_{1}-2\right)\left(3 k_{2}-2\right)+2 k_{2}-1-r} q^{r}\right)^{|\mathbb{C}|}
$$

A substantially better upper bound is provided by the next proposition which makes use of the generalized Fu and Koutras upper bound given in Boutsikas and Koutras [3].

Proposition 4 The cumulative probability $P(S \leq r-1)=R_{r, k_{1}, k_{2}, n_{1}, n_{2}}$ of the scan statistic $S$ is bounded above by

$$
U_{F K}^{(G)}=\prod_{i=1}^{N_{1}-1} \prod_{j=1}^{N_{2}-1}\left(1-P_{i j} Q_{1}\right) \prod_{i=1}^{N_{1}-1}\left(1-P_{i, N_{2}} Q_{2}\right) \prod_{j=1}^{N_{2}-1}\left(1-P_{N_{1}, j} Q_{3}\right)\left(1-P_{N_{1}, N_{2}} Q_{4}\right)
$$

where $Q_{i}, i=1,2,3,4$ are given as in Proposition 2, and

$$
P_{i j}=R\left(\mathbb{C}_{i j}^{* *}\right)=E\left(\prod_{C \in \mathbb{C}_{i j}^{* *}}\left(1-\prod_{(a, b) \in C} X_{a b}\right)\right) .
$$

The quantities $\mathbb{C}_{i j}^{* *}$ are defined as

$$
\mathbb{C}_{i j}^{* *}=\operatorname{minimal}\left\{C \backslash A_{i j}: C \cap A_{i j} \neq \emptyset, C \in \cup_{(a, b)<(i, j)} \mathbb{C}_{a b}\right\},
$$

where $A_{i j}$ are defined in (6) and minimal $(\mathbb{A})$ denotes the set of all minimal sets of $\mathbb{A}$ with respect to the partial ordering induced by the inclusion operator $\subset$.

Proof. Recalling once more the partition described in the proof of Proposition 2 and applying Corollary 1 of Boutsikas and Koutras [3] (the optimum choice of the required binary functions $a_{s}$ should be invoked) we readily obtain the upper bound

$$
U_{F K}^{(G)}=\prod_{i=1}^{N_{1}} \prod_{j=1}^{N_{2}}\left(1-R\left(\mathbb{C}_{i j}^{* *}\right)\left(1-R\left(\mathbb{C}_{i j}\right)\right)\right) .
$$

The evaluation of the quantities $R\left(\mathbb{C}_{i j}\right)$ has already been discussed in Proposition 2.

Since the evaluation of $P_{i j}$ is in general tedious, a convenient lower bound for these quantities would be quite helpful. Such a bound can be easily constructed if we observe that, on introducing the index sets,

$$
\begin{aligned}
K_{i j}= & \left\{(u, v): u=\max \left\{i-k_{1}+1,1\right\}, \ldots, i-1, v=\max \left\{j-k_{2}+1,1\right\}, \ldots, \min \left\{j+2 k_{2}-2, n_{2}\right\}\right\} \\
& \cup\left\{(u, v): u=i, \ldots, i+k_{1}-1, v=\max \left\{j-k_{2}+1,1\right\}, \ldots, j-1\right\} \\
& \cup\left\{(u, v): u=i, \ldots, i+k_{1}-2, v=j+k_{2}, \ldots, \min \left\{j+2 k_{2}-2, n_{2}\right\}\right\}
\end{aligned}
$$


we may write the inequality

$$
\prod_{C \in \mathbb{C}_{i j}^{* * *}}\left(1-\prod_{(u, v) \in C}\left(1-X_{u v}^{\prime}\right)\right) \geq \prod_{(u, v) \in K_{i j}} X_{u v}^{\prime}
$$

which guarantees the validity of the inequality

$$
P_{i j} \geq \prod_{(u, v) \in K_{i j}} p=p^{\left|K_{i j}\right|}
$$

Therefore, it can be easily verified that $P_{i j} \geq p^{\left(k_{1}-1\right)\left(3 k_{2}-2\right)+k_{1}\left(k_{2}-1\right)+\left(k_{1}-1\right)\left(k_{2}-1\right)}$ while a more detailed investigation reveals the following Proposition.

Proposition 5 The cumulative probability $P(S \leq r-1)=R_{r, k_{1}, k_{2}, n_{1}, n_{2}}$ of the scan statistic $S$ is bounded above by

$$
\begin{aligned}
U_{F K}^{(G)} \leq & \left(1-Q_{1}\right)\left(1-p^{\left(k_{1}-1\right)\left(3 k_{2}-2\right)+\left(2 k_{1}-1\right)\left(k_{2}-1\right)} Q_{1}\right)^{\left(n_{1}-k_{1}-1\right)\left(n_{2}-k_{2}-1\right)}\left(1-p^{k_{1}\left(k_{2}-1\right)} Q_{1}\right)^{n_{2}-k_{2}-1} \\
& \times\left(1-p^{\left(k_{1}-1\right)\left(2 k_{2}-1\right)+\left(k_{1}-1\right)\left(k_{2}-1\right)} Q_{1}\right)^{n_{1}-k_{1}-1}\left(1-p^{\left(k_{1}-1\right)\left(2 k_{2}-1\right)+k_{1}\left(k_{2}-1\right)} Q_{2}\right)^{n_{1}-k_{1}} \\
& \times\left(1-p^{\left(k_{1}-1\right)\left(3 k_{2}-2\right)+k_{1}\left(k_{2}-1\right)+\left(k_{1}-1\right)\left(k_{2}-1\right)} Q_{3}\right)^{n_{2}-k_{2}}\left(1-p^{\left(k_{1}-1\right)\left(2 k_{2}-1\right)+k_{1}\left(k_{2}-1\right)} Q_{4}\right)
\end{aligned}
$$

where $Q_{i}, i=1,2,3,4$ are given in Proposition 2 .

For small $r$ 's, the RHS and the LHS of formula (36) are close together while for large $r$ 's the actual value of $P_{i j}$ exhibits strong deviations from the RHS term. Consequently, the upper bound in (37) is sharp only when $r$ is relatively small. A better bound could be established though by conducting a careful inspection of the set $\mathbb{C}_{i j}^{* *}$ for specific values of $k_{1}, k_{2}, r$. For example, for $r=2$, we may derive the following slightly better bound

$$
\begin{aligned}
U_{F K}^{(G) \leq} & \left(1-Q_{1}\right)\left(1-p^{\left(k_{1}-1\right)\left(3 k_{2}-2\right)+k_{1}\left(k_{2}-1\right)} Q_{1}\right)^{\left(n_{1}-k_{1}-1\right)\left(n_{2}-k_{2}-1\right)}\left(1-p^{k_{1}\left(k_{2}-1\right)} Q_{1}\right)^{n_{2}-k_{2}-1} \\
& \times\left(1-p^{\left(k_{1}-1\right)\left(2 k_{2}-1\right)} Q_{1}\right)^{n_{1}-k_{1}-1}\left(1-p^{\left(k_{1}-1\right)\left(2 k_{2}-1\right)+k_{1}\left(k_{2}-1\right)} Q_{2}\right)^{n_{1}-k_{1}} \\
& \times\left(1-p^{\left(k_{1}-1\right)\left(3 k_{2}-2\right)+k_{1}\left(k_{2}-1\right)} Q_{3}\right)^{n_{2}-k_{2}}\left(1-p^{\left(k_{1}-1\right)\left(2 k_{2}-1\right)+k_{1}\left(k_{2}-1\right)} Q_{4}\right)
\end{aligned}
$$

\section{Covariance bounds}

In this Section we are going to develop an upper bound different in nature than the bounds developed so far. The key tool for the construction of this bound is a general result given by Boutsikas and Koutras [3]; since the latter involves covariances of appropriate discrete random variables, the resulting upper bound will be referred as covariance bound. To start with, let us denote by $B_{a b}$ the set of all components $(i, j)$ such that $(i, j)<(a, b)$ (use lexicographic ordering) and $A_{i j} \cap A_{a b} \neq \emptyset$ (so that $R\left(\mathbb{C}_{i j} \cup \mathbb{C}_{a b}\right)=R\left(\mathbb{C}_{i j}\right) R\left(\mathbb{C}_{a b}\right)$ for $\left.(i, j)<(a, b),(i, j) \notin B_{a b}\right)$. It can be easily verified that $B_{a b}$ can be decomposed as $B_{a b}=\cup_{i=1}^{4} B_{a b}^{i}$ where

$$
\begin{aligned}
& B_{a b}^{1}=\left\{(i, j): i=\max \left\{1, a-k_{1}+1\right\}, \ldots, a-1, j=\max \left\{1, b-k_{2}+1\right\}, \ldots, b-1\right\}, \\
& B_{a b}^{2}=\left\{(a, j): j=\max \left\{1, b-k_{2}+1\right\}, \ldots, b-1\right\}, \\
& B_{a b}^{3}=\left\{(i, b): i=\max \left\{1, a-k_{1}+1\right\}, \ldots, a-1\right\}, \\
& B_{a b}^{4}=\left\{(i, j): i=\max \left\{1, a-k_{1}+1\right\}, \ldots, a-1, j=b+1, \ldots, \min \left\{N_{1}, b+k_{2}-1\right\}\right\} .
\end{aligned}
$$

$a=1,2, \ldots, N_{1}, b=1,2, \ldots, N_{2}$ (note that $B_{a b}^{i}$ may occasionally be empty). Finally, let us denote by $F(x, y, z)$ the sum

$$
F(x, y, z)=\sum_{w=0}^{z} F_{r-w, x-z}^{c} F_{r-w, y-z}^{c}\left(\begin{array}{c}
z \\
w
\end{array}\right) q^{w} p^{z-w},
$$

and by $\mathbf{i}$ the quadruple $\left(i_{1}, i_{2}, i_{3}, i_{4}\right)$. 
Proposition 6 The cumulative probability $P(S \leq r-1)=R_{r, k_{1}, k_{2}, n_{1}, n_{2}}$ of the scan statistic $S$ is bounded above by

$$
U_{C B}^{\prime(G)}=\left(1-Q_{1}\right)^{N_{1} N_{2}}+\sum_{m=1}^{4} S_{m}
$$

where $Q_{1}$ is given as in Proposition 2,

$$
\begin{aligned}
& S_{j}=\sum_{a=a_{0}}^{k_{2}-1} \sum_{b=b_{0}}^{k_{1}-1}\left(N_{1}-k_{1}+b\right)\left(N_{2}-k_{2}+a\right)\left(\sum_{\mathbf{i} \in\{0,1\}^{4}}(-1)^{\Sigma_{k=1}^{4} i_{k}} F_{\mathbf{i}}^{(j)}(a, b)-Q_{1}^{2}\right), j=1,4, \\
& S_{2}=\sum_{a=a_{0}}^{k_{2}-1} N_{1}\left(N_{2}-k_{2}+a\right)\left(\sum_{\mathbf{i} \in\{0,1\}^{4}}(-1)^{\Sigma_{k=1}^{4} i_{k}} F_{\mathbf{i}}^{(2)}(a)-Q_{1}^{2}\right), \\
& S_{3}=\sum_{b=b_{0}}^{k_{1}-1} N_{2}\left(N_{1}-k_{1}+b\right)\left(\sum_{\mathbf{i} \in\{0,1\}^{4}}(-1)^{\Sigma_{k=1}^{4} i_{k}} F_{\mathbf{i}}^{(3)}(b)-Q_{1}^{2}\right)
\end{aligned}
$$

with

$$
a_{0}=\max \left\{2 k_{2}-n_{2}, 1\right\}, b_{0}=\max \left\{2 k_{1}-n_{1}, 1\right\},
$$

and

$$
\begin{aligned}
F_{\mathbf{i}}^{(1)}(a, b)= & F\left(\left(k_{1}-i_{2}\right)\left(k_{2}-i_{1}\right)-i_{3} b-i_{4} a+i_{3} i_{4},\left(k_{1}-i_{4}\right)\left(k_{2}-i_{3}\right),\left(a-i_{3}\right)\left(b-i_{4}\right)\right) \\
& \times p^{i_{1} k_{1}+i_{2} k_{2}-i_{1} i_{2}+i_{3} k_{1}+i_{4} k_{2}-i_{3} i_{4}} \\
F_{\mathbf{i}}^{(2)}(a)= & F\left(\left(k_{1}-i_{2}\right)\left(k_{2}-i_{1}-i_{3}\right)-i_{4}\left(1-i_{2}\right)\left(a-i_{3}\right),\left(k_{1}-i_{4}\right)\left(k_{2}-i_{3}\right)-i_{2}\left(1-i_{4}\right)\left(a-i_{3}\right),\right. \\
& \left.,\left(k_{1}-i_{2}\right)\left(a-i_{3}\right)-i_{4}\left(1-i_{2}\right)\left(a-i_{3}\right)\right) \\
& \times p^{i_{1} k_{1}+i_{2} k_{2}-i_{1} i_{2}+i_{3} k_{1}+i_{4} k_{2}-i_{3} i_{4}-i_{2} i_{3}-i_{2} i_{4} a+i_{2} i_{3} i_{4}} \\
F_{\mathbf{i}}^{(3)}(b)= & F\left(\left(k_{2}-i_{2}\right)\left(k_{1}-i_{1}-i_{3}\right)-i_{4}\left(1-i_{2}\right)\left(b-i_{3}\right),\left(k_{2}-i_{4}\right)\left(k_{1}-i_{3}\right)-i_{2}\left(1-i_{4}\right)\left(b-i_{3}\right),\right. \\
& \left.,\left(k_{2}-i_{2}\right)\left(b-i_{3}\right)-i_{4}\left(1-i_{2}\right)\left(b-i_{3}\right)\right) \\
& \times p^{i_{1} k_{2}+i_{2} k_{1}-i_{1} i_{2}+i_{3} k_{2}+i_{4} k_{1}-i_{3} i_{4}-i_{2} i_{3}-i_{2} i_{4} b+i_{2} i_{3} i_{4}}, \\
F_{\mathbf{i}}^{(4)}(a, b)= & F\left(\left(k_{1}-i_{2}\right)\left(k_{2}-i_{1}\right)-i_{3}\left(b-i_{2}\right),\left(k_{1}-i_{4}\right)\left(k_{2}-i_{3}\right)-i_{2}\left(a-i_{3}\right),\left(a-i_{3}\right)\left(b-i_{2}\right)\right) \\
& \times p^{i_{1} k_{1}+i_{2} k_{2}-i_{1} i_{2}+i_{3} k_{1}+i_{4} k_{2}-i_{3} i_{4}-i_{2} i_{3}} .
\end{aligned}
$$

Proof. Applying Theorem 3 of Boutsikas and Koutras [3] for the partition $\mathcal{C}_{1}=\left\{\mathbb{C}_{i j}, i=1,2, \ldots, N_{1}, j=\right.$ $\left.1,2, \ldots, N_{2}\right\}$ of $\mathbb{C}$, we deduce an upper bound of the form

$$
U_{C B}^{(G)}=L_{E P}^{(G)}+\sum_{a=1}^{N_{1}} \sum_{b=1}^{N_{2}} \sum_{(i, j) \in B_{a b}}\left(R\left(\mathbb{C}_{i j} \cup \mathbb{C}_{a b}\right)-R\left(\mathbb{C}_{i j}\right) R\left(\mathbb{C}_{a b}\right)\right) .
$$

Since $\mathcal{C}_{1}$ consists of 4 different types of sets with similar structure (cf. (8)), the final form of the bound (50) will be rather cumbersome. In order to gain a simpler (and slightly worse of course) upper bound we consider the system whose family of minimal cut sets is given by $\mathbb{C}^{\prime}=\bigcup_{i=1}^{N_{1}} \bigcup_{j=1}^{N_{2}} \mathbb{C}_{i j}^{\prime}$ where

$$
\mathbb{C}_{i j}^{\prime}=\mathbb{A}_{i j} \backslash\left(\mathbb{A}_{i+1, j} \cup \mathbb{A}_{i, j+1}\right), i=1,2, \ldots, N_{1} \text { and } j=1,2, \ldots, N_{2} .
$$

Let us also denote by $R_{r, k_{1}, k_{2}, n_{1}, n_{2}}^{\prime}$ the reliability of this last system. Since $\mathbb{C}_{i j}^{\prime}=\mathbb{C}_{i j}$ for $i<N_{1}, j<N_{2}$ and $\mathbb{C}_{i j}^{\prime} \subseteq \mathbb{C}_{i j}$ for $i=N_{1}$ or $j=N_{2}$, it is obvious that $R_{r, k_{1}, k_{2}, n_{1}, n_{2}} \leq R_{r, k_{1}, k_{2}, n_{1}, n_{2}}^{\prime}$. Applying once more 
Theorem 3 of Boutsikas and Koutras [3] for the partition $\mathcal{C}_{2}=\left\{\mathbb{C}_{i j}^{\prime}, i=1,2, \ldots, N_{1}, j=1,2, \ldots, N_{2}\right\}$ we may easily arrive at the upper bound for $R_{r, k_{1}, k_{2}, n_{1}, n_{2}}^{\prime}$ (which is also an upper bound for $R_{r, k_{1}, k_{2}, n_{1}, n_{2}}$ ),

$$
U_{C B}^{\prime(G)}=\prod_{a=1}^{N_{1}} \prod_{b=1}^{N_{2}} R\left(\mathbb{C}_{a b}^{\prime}\right)+\sum_{a=1}^{N_{1}} \sum_{b=1}^{N_{2}} \sum_{(i, j) \in B_{a b}}\left(R\left(\mathbb{C}_{i j}^{\prime} \cup \mathbb{C}_{a b}^{\prime}\right)-R\left(\mathbb{C}_{i j}^{\prime}\right) R\left(\mathbb{C}_{a b}^{\prime}\right)\right)
$$

where, in this case, $R\left(\mathbb{C}_{a b}^{\prime}\right)=1-Q_{1}$. On setting $\delta(\mathbb{A})=\prod_{A \in \mathbb{A}}\left(1-\prod_{i \in A}\left(1-X_{i}^{\prime}\right)\right)$, the triple sum appearing in $U_{C B}^{\prime(G)}$ takes on the form

$$
\begin{aligned}
\sum_{a=1}^{N_{1}} \sum_{b=1}^{N_{2}} \sum_{(i, j) \in B_{a b}} \operatorname{Cov}\left(\delta\left(\mathbb{C}_{i j}^{\prime}\right), \delta\left(\mathbb{C}_{a b}^{\prime}\right)\right) & =\sum_{a=1}^{N_{1}} \sum_{b=1}^{N_{2}} \sum_{(i, j) \in B_{a b}} \operatorname{Cov}\left(1-\delta\left(\mathbb{C}_{i j}^{\prime}\right), 1-\delta\left(\mathbb{C}_{a b}^{\prime}\right)\right) \\
& =\sum_{a=1}^{N_{1}} \sum_{b=1}^{N_{2}} \sum_{(i, j) \in B_{a b}}\left(P\left(\delta\left(\mathbb{C}_{i j}^{\prime}\right)=0, \delta\left(\mathbb{C}_{a b}^{\prime}\right)=0\right)-Q_{1}^{2}\right)=\sum_{m=1}^{4} S_{m}
\end{aligned}
$$

where

$$
S_{m}=\sum_{a=1}^{N_{1}} \sum_{b=1}^{N_{2}} \sum_{(i, j) \in B_{a b}^{m}}\left(P\left(\delta\left(\mathbb{C}_{a b}^{\prime}\right)=0, \delta\left(\mathbb{C}_{i j}^{\prime}\right)=0\right)-Q_{1}^{2}\right) .
$$

For the evaluation of $S_{1}$ we observe that

$$
S_{1}=\sum_{a=a_{0}}^{k_{2}-1} \sum_{b=b_{0}}^{k_{1}-1}\left(N_{1}-k_{1}+b\right)\left(N_{2}-k_{2}+a\right)\left(P\left(\delta\left(\mathbb{C}_{11}^{\prime}\right)=0, \delta\left(\mathbb{C}_{k_{1}-b+1, k_{2}-a+1}^{\prime}\right)=0\right)-Q_{1}^{2}\right) ;
$$

the computation of $P\left(\delta\left(\mathbb{C}_{11}^{\prime}\right)=0, \delta\left(\mathbb{C}_{k_{1}-b+1, k_{2}-a+1}^{\prime}\right)=0\right)$ can be carried out by expressing the event of interest as intersection of events $A B_{1}^{c} B_{2}^{c} B_{3}^{c} B_{4}^{c}$ where

$$
A=W_{11} W_{k_{1}-b+1, k_{2}-a+1}, \quad B_{1}=V_{11}, \quad B_{2}=H_{11}, \quad B_{3}=V_{k_{1}-b+1, k_{2}-a+1}, \quad B_{4}=H_{k_{1}-b+1, k_{2}-a+1} .
$$

More specifically, by virtue of the inclusion-exclusion theorem we may write

$$
P\left(A \cap\left(\bigcap_{i=1}^{4} B_{i}^{c}\right)\right)=P(A)-P\left(\bigcup_{i=1}^{4} A B_{i}\right)=P(A)-\sum_{s=1}^{4} \sum_{D \subseteq\{1,2,3,4\}:|D|=s}(-1)^{s-1} P\left(\bigcap_{i \in D} A B_{i}\right)
$$

and the evaluation of the probabilities $P\left(\cap_{i \in D} A B_{i}\right)$ can be accomplished if we recognize that the events of interest can be expressed via iid binary r.v.'s $Z_{i}$ such that $P\left(Z_{i}=1\right)=q$. In particular, it is not difficult to check that, on setting

$$
\begin{aligned}
F(x, y, z) & =P\left(\sum_{i=1}^{x} Z_{i} \geq r, \sum_{i=x-z+1}^{x-z+y} Z_{i} \geq r\right) \\
& =\sum_{w=0}^{z} P\left(\sum_{i=1}^{x-z} Z_{i} \geq r-w, \sum_{i=x+1}^{x-z+y} Z_{i} \geq r-w\right) P\left(\sum_{i=x-z+1}^{x} Z_{i}=w\right)
\end{aligned}
$$

(a convenient expression for that is given in (40)) we may write $P\left(\cap_{i \in D} A B_{i}\right)=F_{\mathbf{i}}^{(1)}(a, b), \mathbf{i}=\left(i_{1}, i_{2}, i_{3}, i_{4}\right)$ with the indices $i_{j}, j=1,2,3,4$ being 1 or 0 according to whether $j \in D$ or $j \notin D$ respectively and

$$
\begin{aligned}
F_{\mathbf{i}}^{(1)}(a, b)= & F_{\mathbf{i}}^{(1)}\left(a, b ; k_{1}, k_{2}\right)=p^{i_{1} k_{1}+i_{2} k_{2}-i_{1} i_{2}+i_{3} k_{1}+i_{4} k_{2}-i_{3} i_{4}} \times \\
& \times F\left(\left(k_{1}-i_{2}\right)\left(k_{2}-i_{1}\right)-i_{3} b-i_{4} a+i_{3} i_{4},\left(k_{1}-i_{4}\right)\left(k_{2}-i_{3}\right),\left(a-i_{3}\right)\left(b-i_{4}\right)\right) .
\end{aligned}
$$


Combining all the above we deduce

$$
S_{1}=\sum_{a=a_{0}}^{k_{2}-1} \sum_{b=b_{0}}^{k_{1}-1}\left(N_{1}-k_{1}+b\right)\left(N_{2}-k_{2}+a\right)\left(\sum_{\mathbf{i} \in\{0,1\}^{4}}(-1)^{\Sigma_{k=1}^{4} i_{k}} F_{\mathbf{i}}^{(1)}(a, b)-Q_{1}^{2}\right) .
$$

Arguing in exactly the same way, we may also compute $S_{2}, S_{3}, S_{4}$ (the technical details are lengthy but straightforward).

\section{Asymptotic results}

Many real life applications call for the study of the two dimensional scan statistic $S$ in a large square grid of size $n_{1} \times n_{2}$ (i.e. for large $n_{1}, n_{2}$ ) or equivalently, for the study of huge two-dimensional $r$-withinconsecutive- $k_{1} \times k_{2}$-out-of- $n_{1} \times n_{2}$ reliability structures with high quality components. In these cases, the evaluation of lower and upper bounds though feasible, quickly become tedious. An asymptotic estimate of the tail probability or system's reliability, may therefore be useful. In this paragraph we aim at an asymptotic result concerning the case when $n_{1}, n_{2}$ converge to infinity and at the same time $q \rightarrow 0$ so that $n_{1} n_{2} q^{r}$ converges to a positive number. To achieve this we may use either the bounds developed in the previous section (e.g. $L_{E P}, U_{F K}$ ) or the general results for coherent structures presented by $\mathrm{Fu}$ and Koutras [7].

Proposition 7 If $r, k_{1}, k_{2}$ are fixed and $n_{1}, n_{2} \rightarrow \infty, q \rightarrow 0$ such that

$$
n_{1} n_{2}\left(\left(\begin{array}{c}
k_{1} k_{2} \\
r
\end{array}\right)-\left(\begin{array}{c}
\left(k_{1}-1\right) k_{2} \\
r
\end{array}\right)-\left(\begin{array}{c}
k_{1}\left(k_{2}-1\right) \\
r
\end{array}\right)+\left(\begin{array}{c}
\left(k_{1}-1\right)\left(k_{2}-1\right) \\
r
\end{array}\right)\right) q^{r} \rightarrow \lambda \in(0, \infty)
$$

then

$$
R_{r, k_{1}, k_{2}, n_{1}, n_{2}} \rightarrow e^{-\lambda} .
$$

Proof. It suffices to observe that $\max \left|L_{C_{j}}\right|$ remains bounded as $n_{1}, n_{2} \rightarrow \infty$ (cf. 28) and then apply Corollary 4.2. of Fu and Koutras [7]. The desired result follows immediately, on using expression (14) for $|\mathbb{C}|$. An alternative proof of the same result can also be established by using the simpler bounds $L_{E P}, U_{F K}$ developed in the previous sections.

We are now presenting an interesting consequence of the above proposition relating to the asymptotic distribution of the time to failure $T$ of a two-dimensional $r$-within-consecutive- $k_{1} \times k_{2}$-out-of- $n_{1} \times n_{2}$ reliability system. More specifically, suppose that this system starts functioning at time 0 equipped with $n_{1} n_{2}$ new (unused) components and denote by $T$ the time until system's failure and by $T_{i j}$ the lifetime of the $(i, j)$ component. Then we have the next result.

Corollary 1 Let the common lifetime distributions of the components be of the form

$$
P\left(T_{i j} \leq t\right)=q(t)=\theta^{a} t^{a}+o\left(t^{a}\right), t \geq 0
$$

where $a, \theta$ are positive constants. Then, the asymptotic lifetime $T$ of the system (properly normalized) follows a Weibull distribution with parameter

$$
\theta^{\prime}=\theta\left(\left(\begin{array}{c}
k_{1} k_{2} \\
r
\end{array}\right)-\left(\begin{array}{c}
\left(k_{1}-1\right) k_{2} \\
r
\end{array}\right)-\left(\begin{array}{c}
k_{1}\left(k_{2}-1\right) \\
r
\end{array}\right)+\left(\begin{array}{c}
\left(k_{1}-1\right)\left(k_{2}-1\right) \\
r
\end{array}\right)\right)^{1 / a r},
$$

more specifically,

$$
P\left(\left(n_{1} n_{2}\right)^{1 / r a} T>t\right)=R\left(\left(n_{1} n_{2}\right)^{-1 / r a} t\right) \rightarrow e^{-\left(\theta^{\prime} t\right)^{a r}}
$$

as $n_{1}, n_{2} \rightarrow \infty$. 
Proof. It is an immediate consequence of Proposition 7 since $q=q\left(\left(n_{1} n_{2}\right)^{-1 / r a} t\right) \rightarrow 0$ and

$$
\begin{aligned}
n_{1} n_{2} q^{r} & =n_{1} n_{2} q\left(\left(n_{1} n_{2}\right)^{-1 / r a} t\right)^{r}=n_{1} n_{2}\left(\theta^{a}\left(n_{1} n_{2}\right)^{-1 / r} t^{a}+o\left(\left(n_{1} n_{2}\right)^{-1 / r}\right)\right)^{r} \\
& =\left(\theta^{a} t^{a}+\frac{o\left(\left(n_{1} n_{2}\right)^{-1 / r}\right)}{\left(n_{1} n_{2}\right)^{-1 / r}}\right)^{r} \rightarrow(\theta t)^{a r}
\end{aligned}
$$

as $n_{1}, n_{2} \rightarrow \infty$.

Note that, the assumption made above that components' failure distribution satisfies $q(t)=\theta^{a} t^{a}+$ $o\left(t^{a}\right), t \geq 0$, is a rather mild one (it is equivalent to $\lim _{t \rightarrow 0^{+}} t^{-a} q(t) \in(0, \infty)$ for some $a>0$ ). There are quite a few lifetime distributions which satisfy this condition, including exponential, Weibull, gamma, uniform, etc.

\section{Numerical comparisons}

In Tables 1-9 we provide, for selected values of $r, k_{1}, k_{2}, n_{1}, p$, the values of the bounds described in formulae (13), (18), (29), (37) (or (38) for $r=2$ ), (41) (they have been labeled as $L_{E P}, L_{E P}^{G}, U_{F K}, U_{F K}^{G}, U_{C B}^{G}$ respectively) along with the Bonferroni-type bounds developed by Makri and Psillakis [9] (for the case $\left.n_{1}=n_{2}, k_{1}=k_{2}\right)$. These bounds have been labeled as $L_{\text {Bonf }}, U_{B o n f}$. We also provide a simulated value $\left(R_{\text {simul }}\right)$ and an approximate value $\left(R_{\text {approx }}\right)$ based on the results of Boutsikas and Koutras [4].

It is worth stressing that for large systems (e.g. $\left.n_{1}, n_{2}>30\right)$, the computer times for assessing the simulated values of $R$ are extremely large, especially when a large number of iterations are performed. This is why in most cases we were forced to reduce the number of iterations to $10^{4}$ or $10^{5}$ (only in Table 1 we employ $10^{6}$ iterations). This fact may have resulted in quite crude approximations of the exact reliability. However, the bounds are easily evaluated no matter how large the values of $n, k$ are.

A careful inspection of the tables reveals that $L_{E P}^{G}$ is the best lower bound of the three for each of the cases we consider, while, for very high component survival probabilities, $U_{C B}^{G}$ is the best of the four upper bounds. For lower component survival probabilities the leading bounds are $U_{F K}^{G}$ (for small $r$ ) or $U_{\text {Bonf }}$.

\section{References}

[1] Akiba and Yamamoto (2001). Reliability of a two-dimensional $k$-within-consecutive- $r \times s$-out-of$m \times n: F$ system. Naval Research Logistics 48, 625-637.

[2] Barlow, R.E. and Proschan, F. (1981). Statistical Theory of Reliability and Life Testing. To Begin with, Silver Spring, MD.

[3] Boutsikas, M.V. and Koutras, M.V. (2000). Generalized reliability bounds for coherent structures. Journal of Applied Probability 37, 778-794.

[4] Boutsikas M.V. and Koutras M.V. (2000). Reliability Approximation for Markov Chain Imbeddable Systems. Methodology and Computing in Applied Probability 2:4, 393-411.

[5] Chen, J. and Glaz, J. (1996). Two-dimensional discrete scan statistics. Statistics and Probability Letters 31, 59-68.

[6] Esary, J.D. and Proschan, F. (1963) Coherent structures of non-identical components. Technometrics 5, 191-209.

[7] Fu, J.C. and Koutras, M.V. (1995). Reliability bounds for coherent structures with independent components. Statistics \& Probability Letters 22, 137-148. 
[8] Glaz, J., Naus J. and Wallenstein S. (2001) Scan Statistics. Springer - Verlag.

[9] Makri, F.S. and Psillakis, Z.M. (1997). Bounds for reliability of $k$-within 2-dimensional consecutive$r$-out-of- $n$ failure systems. Microelectronics and Reliability 37, 1217-1224.

[10] Orford, K.J. (2000) The analysis of cosmic ray data. Journal of Physics G-Nuclear and Particle Physics 26(4), R1-R26. 
Table 1, $r=2, k_{1}=3, k_{2}=3, n_{1}=30, n_{2}=30$

\begin{tabular}{c|ccc|cc|cccc}
\hline $\boldsymbol{p}$ & $\boldsymbol{L}_{\boldsymbol{E P}}$ & $\boldsymbol{L}_{\boldsymbol{E P}}{ }^{\boldsymbol{G}}$ & $\boldsymbol{L B \text { Bonf }}$ & $\boldsymbol{R}_{\text {approx }}$ & $\boldsymbol{R}_{\text {simul }}$ & $\boldsymbol{U B o n f}$ & $\boldsymbol{U}_{\boldsymbol{F K}}$ & $\boldsymbol{U}_{\boldsymbol{F K}}{ }^{\boldsymbol{G}}$ & $\boldsymbol{U}_{\boldsymbol{C B}}{ }^{\boldsymbol{G}}$ \\
\hline .982 & 0.0402 & $\mathbf{0 . 0 4 7 6}$ & -7.2781 & 0.0777 & 0.0838 & 0.3536 & 0.1604 & $\mathbf{0 . 1 1 5 4}$ & 0.7995 \\
.986 & 0.1431 & $\mathbf{0 . 1 5 5 1}$ & -4.1247 & 0.1980 & 0.2071 & 0.4603 & 0.2849 & $\mathbf{0 . 2 4 0 0}$ & 0.5339 \\
.99 & 0.3709 & $\mathbf{0 . 3 8 2 1}$ & -1.6731 & 0.4199 & 0.4284 & 0.6127 & 0.4837 & $\mathbf{0 . 4 5 1 4}$ & 0.5387 \\
.994 & 0.6997 & $\mathbf{0 . 7 0 4 3}$ & 0.0171 & 0.7197 & 0.7237 & 0.8044 & 0.7436 & $\mathbf{0 . 7 3 1 4}$ & 0.7478 \\
.998 & 0.9611 & $\mathbf{0 . 9 6 1 3}$ & 0.8886 & 0.9622 & 0.9624 & 0.9717 & 0.9634 & $\mathbf{0 . 9 6 2 8}$ & 0.9645 \\
.999 & 0.9901 & $\mathbf{0 . 9 9 0 2}$ & 0.9720 & 0.9903 & 0.9902 & 0.9926 & 0.9904 & $\mathbf{0 . 9 9 0 3}$ & 0.9908 \\
\hline
\end{tabular}

Table 2, $r=3, k_{1}=4, k_{2}=4, n_{1}=100, n_{2}=100$

\begin{tabular}{c|ccc|cc|cccc}
\hline $\boldsymbol{p}$ & $\boldsymbol{L}_{\boldsymbol{E P}}$ & $\boldsymbol{L}_{\boldsymbol{E P}} \boldsymbol{G}$ & $\boldsymbol{L b o n f}$ & $\boldsymbol{R}_{\text {approx }}$ & $\boldsymbol{R}_{\text {simul }}$ & $\boldsymbol{U B o n f}$ & $\boldsymbol{U}_{\boldsymbol{F K}}$ & $\boldsymbol{U}_{\boldsymbol{F K}}{ }^{\boldsymbol{G}}$ & $\boldsymbol{U}_{\boldsymbol{C B}}{ }^{\boldsymbol{G}}$ \\
\hline .99 & 0.1429 & $\mathbf{0 . 1 6 6 9}$ & -3.7749 & 0.2080 & 0.2207 & 0.5003 & 0.3596 & $\mathbf{0 . 3 3 8 3}$ & 0.5403 \\
.992 & 0.3692 & $\mathbf{0 . 3 9 3 7}$ & -1.4936 & 0.4321 & 0.4464 & 0.6486 & 0.5511 & $\mathbf{0 . 5 3 5 9}$ & 0.5497 \\
.994 & 0.6568 & $\mathbf{0 . 6 7 0 4}$ & -0.0729 & 0.6910 & 0.6959 & 0.8033 & 0.7513 & 0.7439 & $\mathbf{0 . 7 2 1 7}$ \\
.996 & 0.8829 & $\mathbf{0 . 8 8 6 5}$ & 0.6758 & 0.8920 & 0.8916 & 0.9281 & 0.9081 & 0.9061 & $\mathbf{0 . 8 9 7 3}$ \\
.998 & 0.9846 & $\mathbf{0 . 9 8 4 8}$ & 0.9587 & 0.9852 & 0.9850 & 0.9899 & 0.9864 & 0.9862 & $\mathbf{0 . 9 8 5 6}$ \\
\hline
\end{tabular}

Table 3, $r=6, k_{1}=3, k_{2}=3, n_{1}=1000, n_{2}=1000$

\begin{tabular}{c|ccc|cc|cccc}
\hline $\boldsymbol{p}$ & $\boldsymbol{L}_{\boldsymbol{E P}}$ & $\boldsymbol{L}_{\boldsymbol{E P}} \boldsymbol{G}^{\boldsymbol{L}}$ & $\boldsymbol{L B O n f}$ & $\boldsymbol{R}_{\text {approx }}$ & $\boldsymbol{R}_{\text {simul }}$ & $\boldsymbol{U B o n f}$ & $\boldsymbol{U}_{\boldsymbol{F K}}$ & $\boldsymbol{U}_{\boldsymbol{F K}}{ }^{\boldsymbol{G}}$ & $\boldsymbol{U}_{\boldsymbol{C B}}{ }^{\boldsymbol{G}}$ \\
\hline .93 & 0.0001 & $\mathbf{0 . 0 0 0 3}$ & -7.1775 & 0.0008 & 0.0009 & $\mathbf{0 . 1 3 4 6}$ & 0.2581 & 0.2451 & 1.0089 \\
.94 & 0.0221 & $\mathbf{0 . 0 3 8 6}$ & -2.3322 & 0.0522 & 0.0518 & $\mathbf{0 . 2 6 4 5}$ & 0.4883 & 0.4772 & 0.3769 \\
.95 & 0.2791 & $\mathbf{0 . 3 2 6 4}$ & -0.1464 & 0.3554 & 0.3614 & 0.4953 & 0.7265 & 0.7205 & $\mathbf{0 . 4 1 9 6}$ \\
.96 & 0.7157 & $\mathbf{0 . 7 3 9 7}$ & 0.6913 & 0.7532 & 0.7545 & 0.7658 & 0.8948 & 0.8928 & $\mathbf{0 . 7 5 9 1}$ \\
.97 & 0.9422 & $\mathbf{0 . 9 4 6 4}$ & 0.9436 & 0.9487 & 0.9470 & 0.9491 & 0.9742 & 0.9738 & $\mathbf{0 . 9 4 9 0}$ \\
.98 & 0.9948 & $\mathbf{0 . 9 9 5 0}$ & 0.9949 & 0.9952 & 0.9951 & 0.9952 & 0.9970 & 0.9969 & $\mathbf{0 . 9 9 5 2}$ \\
\hline
\end{tabular}

Table 4, $r=12, k_{1}=4, k_{2}=4, n_{1}=100, n_{2}=100$

\begin{tabular}{c|ccc|cc|cccc}
\hline $\boldsymbol{p}$ & $\boldsymbol{L}_{\boldsymbol{E P}}$ & $\boldsymbol{L}_{\boldsymbol{E P}} \boldsymbol{G}$ & $\boldsymbol{L B o n f}$ & $\boldsymbol{R}_{\text {approx }}$ & $\boldsymbol{R}_{\text {simul }}$ & $\boldsymbol{U B O n f}$ & $\boldsymbol{U}_{\boldsymbol{F K}}$ & $\boldsymbol{U}_{\boldsymbol{F K}}{ }^{\boldsymbol{G}}$ & $\boldsymbol{U}_{\boldsymbol{C B}}{ }^{\boldsymbol{G}}$ \\
\hline .7 & 0.0001 & $\mathbf{0 . 0 8 2 2}$ & -1.5003 & 0.1777 & 0.1862 & $\mathbf{0 . 4 4 6 2}$ & 1. & 0.9994 & 1.4161 \\
.72 & 0.0188 & $\mathbf{0 . 2 9 9 2}$ & -0.2075 & 0.4164 & 0.4265 & $\mathbf{0 . 6 0 6 7}$ & 1. & 0.9996 & 0.8312 \\
.74 & 0.1955 & $\mathbf{0 . 5 7 8 9}$ & 0.4529 & 0.6603 & 0.6615 & $\mathbf{0 . 7 5 1 5}$ & 1. & 0.9998 & 0.7769 \\
.76 & 0.5354 & $\mathbf{0 . 7 9 4 4}$ & 0.7697 & 0.8336 & 0.8352 & 0.8647 & 1. & 0.9999 & $\mathbf{0 . 8 6 2 5}$ \\
.78 & 0.8026 & $\mathbf{0 . 9 1 4 9}$ & 0.911 & 0.9297 & 0.9292 & 0.9364 & 1. & 0.9999 & $\mathbf{0 . 9 3 6 3}$ \\
.8 & 0.9323 & $\mathbf{0 . 9 6 9 4}$ & 0.9689 & 0.9740 & 0.9737 & 0.9755 & 1. & 1. & $\mathbf{0 . 9 7 5 4}$ \\
.82 & 0.9804 & $\mathbf{0 . 9 9 0 5}$ & 0.9904 & 0.9917 & 0.9916 & 0.9919 & 1. & 1. & $\mathbf{0 . 9 9 1 9}$ \\
\hline
\end{tabular}

Table 5, $r=5, k_{1}=5, k_{2}=5, n_{1}=100, n_{2}=100$

\begin{tabular}{c|ccc|cc|cccc}
\hline $\boldsymbol{p}$ & $\boldsymbol{L}_{\boldsymbol{E P}}$ & $\boldsymbol{L}_{\boldsymbol{E P}} \boldsymbol{G}$ & $\boldsymbol{L B o n f}$ & $\boldsymbol{R}_{\text {approx }}$ & $\boldsymbol{R}_{\text {simul }}$ & $\boldsymbol{U B \text { Bonf }}$ & $\boldsymbol{U}_{\boldsymbol{F K}}$ & $\boldsymbol{U}_{\boldsymbol{F K}}{ }^{\boldsymbol{G}}$ & $\boldsymbol{U}_{\boldsymbol{C B}}^{\boldsymbol{G}}$ \\
\hline .97 & 0.0025 & $\mathbf{0 . 0 2 4 6}$ & -6.1884 & 0.0755 & 0.0967 & $\mathbf{0 . 4 2 9 2}$ & 0.8001 & 0.7533 & 2.4923 \\
.975 & 0.0903 & $\mathbf{0 . 1 9 9 3}$ & -2.1440 & 0.3018 & 0.3331 & $\mathbf{0 . 6 0 8 8}$ & 0.8554 & 0.8288 & 1.0415 \\
.98 & 0.4547 & $\mathbf{0 . 5 6 4 1}$ & -0.1208 & 0.6361 & 0.6543 & 0.7956 & 0.9149 & 0.9030 & $\mathbf{0 . 7 8 9 5}$ \\
.985 & 0.8294 & $\mathbf{0 . 8 6 3 1}$ & 0.7107 & 0.8838 & 0.8888 & 0.9313 & 0.9641 & 0.9605 & $\mathbf{0 . 9 0 4 2}$ \\
.99 & 0.9757 & $\mathbf{0 . 9 7 9 2}$ & 0.9586 & 0.9815 & 0.9821 & 0.9882 & 0.9917 & 0.9912 & $\mathbf{0 . 9 8 2 9}$ \\
.995 & 0.9992 & $\mathbf{0 . 9 9 9 3}$ & 0.9986 & 0.9993 & 0.9993 & 0.9996 & 0.9996 & 0.9995 & $\mathbf{0 . 9 9 9 4}$ \\
\hline
\end{tabular}

Table 6, $r=20, k_{1}=5, k_{2}=5, n_{1}=1000, n_{2}=1000$

\begin{tabular}{c|ccc|cc|cccc}
\hline $\boldsymbol{p}$ & $\boldsymbol{L}_{\boldsymbol{E P}}$ & $\boldsymbol{L}_{\boldsymbol{E P}} \boldsymbol{G}$ & $\boldsymbol{L B o n f}$ & $\boldsymbol{R}_{\text {approx }}$ & $\boldsymbol{R}_{\text {simul }}$ & $\boldsymbol{U B o n f}$ & $\boldsymbol{U}_{\boldsymbol{F K}}$ & $\boldsymbol{U}_{\boldsymbol{F K}} \boldsymbol{G}^{\boldsymbol{G}} \boldsymbol{U}_{\boldsymbol{C B}} \boldsymbol{G}^{\boldsymbol{G}}$ \\
\hline .64 & 0.0000 & $\mathbf{0 . 0 0 0 2}$ & -7.6897 & 0.0014 & 0.0013 & $\mathbf{0 . 1 6 7 3}$ & 1. & 1. & 3.3426 \\
.66 & 0.0000 & $\mathbf{0 . 0 4 1 1}$ & -2.1925 & 0.0809 & 0.0793 & $\mathbf{0 . 3 3 2 8}$ & 1. & 1. & 1.0427 \\
.68 & 0.0013 & $\mathbf{0 . 3 3 6 2}$ & -0.0902 & 0.4106 & 0.4066 & $\mathbf{0 . 5 6 4 1}$ & 1. & 1. & 0.6145 \\
.7 & 0.1592 & $\mathbf{0 . 7 0 9 7}$ & 0.6570 & 0.7489 & 0.7481 & 0.7869 & 1. & 1. & $\mathbf{0 . 7 8 0 7}$ \\
.72 & 0.6298 & $\mathbf{0 . 9 0 6 3}$ & 0.9016 & 0.9182 & 0.9120 & 0.9229 & 1. & 1. & $\mathbf{0 . 9 2 2 7}$ \\
.74 & 0.9003 & $\mathbf{0 . 9 7 4 9}$ & 0.9746 & 0.9777 & 0.9780 & 0.9783 & 1. & 1. & $\mathbf{0 . 9 7 8 3}$ \\
.76 & 0.9790 & $\mathbf{0 . 9 9 4 2}$ & 0.9942 & 0.9947 & 0.9945 & 0.9948 & 1. & 1. & $\mathbf{0 . 9 9 4 8}$ \\
\hline
\end{tabular}


Table 7, $r=2, k_{1}=4, k_{2}=4, n_{1}=500, n_{2}=500$

\begin{tabular}{c|ccc|cc|cccc}
\hline $\boldsymbol{p}$ & $\boldsymbol{L}_{\boldsymbol{E P}}$ & $\boldsymbol{L}_{\boldsymbol{E P}}{ }^{\boldsymbol{G}}$ & $\boldsymbol{L B o n f}$ & $\boldsymbol{R}_{\text {approx }}$ & $\boldsymbol{R}_{\text {simul }}$ & $\boldsymbol{U B o n f}$ & $\boldsymbol{U}_{\boldsymbol{F K}}$ & $\boldsymbol{U}_{\boldsymbol{F K}}{ }^{\boldsymbol{G}}$ & $\boldsymbol{U}_{\boldsymbol{C B}}{ }^{\boldsymbol{G}}$ \\
\hline .9992 & 0.0221 & $\mathbf{0 . 0 2 2 5}$ & -17.827 & 0.0239 & 0.0250 & 0.2750 & 0.0268 & $\mathbf{0 . 0 2 5 4}$ & 0.1312 \\
.9993 & 0.0540 & $\mathbf{0 . 0 5 4 6}$ & -13.428 & 0.0570 & 0.0588 & 0.3301 & 0.0614 & $\mathbf{0 . 0 5 9 4}$ & 0.1281 \\
.9994 & 0.1171 & $\mathbf{0 . 1 1 7 9}$ & -9.6105 & 0.1211 & 0.1223 & 0.4005 & 0.1271 & $\mathbf{0 . 1 2 4 3}$ & 0.1650 \\
.9995 & 0.2255 & $\mathbf{0 . 2 2 6 4}$ & -6.3754 & 0.2300 & 0.2334 & 0.4892 & 0.2365 & $\mathbf{0 . 2 3 3 5}$ & 0.2547 \\
.9996 & 0.3855 & $\mathbf{0 . 3 8 6 3}$ & -3.7247 & 0.3894 & 0.3900 & 0.5985 & 0.3950 & $\mathbf{0 . 3 9 2 4}$ & 0.4018 \\
.9997 & 0.5850 & $\mathbf{0 . 5 8 5 5}$ & -1.6601 & 0.5875 & 0.5893 & 0.7251 & 0.5910 & $\mathbf{0 . 5 8 9 4}$ & 0.5928 \\
.9998 & 0.7879 & $\mathbf{0 . 7 8 8 2}$ & -0.1834 & 0.7890 & 0.7883 & 0.8554 & 0.7904 & $\mathbf{0 . 7 8 9 7}$ & 0.7908 \\
.9999 & 0.9422 & $\mathbf{0 . 9 4 2 2}$ & 0.7039 & 0.9423 & 0.9424 & 0.9592 & 0.9425 & $\mathbf{0 . 9 4 2 4}$ & 0.9427 \\
.99995 & 0.9852 & $\mathbf{0 . 9 8 5 2}$ & 0.9259 & 0.9852 & 0.9852 & 0.9895 & 0.9853 & $\mathbf{0 . 9 8 5 2}$ & 0.9853 \\
\hline
\end{tabular}

Table 8, $r=3, k_{1}=2, k_{2}=5, n_{1}=100, n_{2}=20$

\begin{tabular}{c|cc|cc|ccc}
\hline $\boldsymbol{p}$ & $\boldsymbol{L}_{\boldsymbol{E P}}$ & $\boldsymbol{L}_{\boldsymbol{E P} \boldsymbol{P}}{ }^{\boldsymbol{G}}$ & $\boldsymbol{R}_{\text {approx }}$ & $\boldsymbol{R}_{\text {simul }}$ & $\boldsymbol{U}_{\boldsymbol{F} \boldsymbol{K}}$ & $\boldsymbol{U}_{\boldsymbol{F K}}{ }^{\boldsymbol{G}}$ & $\boldsymbol{U}_{\boldsymbol{C B}}{ }^{\boldsymbol{G}}$ \\
\hline .965 & 0.0155 & $\mathbf{0 . 0 2 9 2}$ & 0.0608 & 0.0678 & 0.2640 & $\mathbf{0 . 2 1 0 6}$ & 1.1422 \\
.97 & 0.0726 & $\mathbf{0 . 1 0 2 6}$ & 0.1549 & 0.1635 & 0.3718 & $\mathbf{0 . 3 2 3 2}$ & 0.7218 \\
.975 & 0.2193 & $\mathbf{0 . 2 5 9 5}$ & 0.3192 & 0.3282 & 0.5092 & $\mathbf{0 . 4 7 1 2}$ & 0.5743 \\
.98 & 0.4598 & $\mathbf{0 . 4 9 3 1}$ & 0.5386 & 0.5476 & 0.6656 & 0.6418 & $\mathbf{0 . 6 3 4 0}$ \\
.985 & 0.7205 & $\mathbf{0 . 7 3 6 8}$ & 0.7585 & 0.7640 & 0.8170 & 0.8063 & $\mathbf{0 . 7 8 7 7}$ \\
.99 & 0.9074 & $\mathbf{0 . 9 1 1 5}$ & 0.9170 & 0.9175 & 0.9300 & 0.9292 & $\mathbf{0 . 9 2 3 7}$ \\
.995 & 0.9879 & $\mathbf{0 . 9 8 8 2}$ & 0.9886 & 0.9888 & 0.9897 & 0.9895 & $\mathbf{0 . 9 8 9 3}$ \\
\hline
\end{tabular}

Table 9, $r=15, k_{1}=6, k_{2}=3, n_{1}=1000, n_{2}=100$

\begin{tabular}{c|cc|cc|ccc}
\hline $\boldsymbol{p}$ & $\boldsymbol{L}_{\boldsymbol{E P}}$ & $\boldsymbol{L}_{\boldsymbol{E} \boldsymbol{P}}{ }^{\boldsymbol{B}}$ & $\boldsymbol{R}_{\text {approx }}$ & $\boldsymbol{R}_{\text {simul }}$ & $\boldsymbol{U}_{\boldsymbol{F K}}$ & $\boldsymbol{U}_{\boldsymbol{F K}}{ }^{\boldsymbol{G}}$ & $\boldsymbol{U}_{\boldsymbol{C B}}{ }^{\boldsymbol{G}}$ \\
\hline .7 & 0.3197 & $\mathbf{0 . 6 5 4 3}$ & 0.7071 & 0.7090 & 1. & 1. & $\mathbf{0 . 7 5 8 1}$ \\
.72 & 0.6669 & $\mathbf{0 . 8 4 9 8}$ & 0.8723 & 0.8718 & 1. & 1. & $\mathbf{0 . 8 8 3 2}$ \\
.74 & 0.8752 & $\mathbf{0 . 9 4 3 9}$ & 0.9515 & 0.9513 & 1. & 1. & $\mathbf{0 . 9 5 3 8}$ \\
.76 & 0.9607 & $\mathbf{0 . 9 8 1 5}$ & 0.9837 & 0.9830 & 1. & 1. & $\mathbf{0 . 9 8 4 2}$ \\
.78 & 0.9892 & $\mathbf{0 . 9 9 4 6}$ & 0.9951 & 0.9949 & 1. & 1. & $\mathbf{0 . 9 9 5 2}$ \\
\hline
\end{tabular}

